Original Research Paper

\title{
Redox Modifications and Hematological Neoplasms-an Overview
}

\author{
Fernanda Carolina de Campos \\ Faculdade Estácio de Sá, Campus de Ourinhos, FAESO, Ourinhos, São Paulo, Brazil
}

Article history

Received: 05-02-2014

Revised: 06-08-2014

Accepted: 05-01-2015

\begin{abstract}
Due to our current interest in the study of the relationship between hematological malignancies and oxidative stress, in this review we addressed the recent finding regarding this issue, focusing on the clinical findings concerning the oxidative status of patients diagnosed with hematological cancers. This study is a descriptive review of the literature. For the theoretical scientific background, we used the electronic PubMed search engines. It is possible to find several studies involving oxidative stress and hematological cancer, analyzing the many ways regarding the imbalance of excessive free radical production and its neutralization by antioxidants. The present review highlights the need for studies to understand the main role of oxidative stress as a cause or consequence of hematological neoplasms, as well as its participation as beneficial for either the host or tumor cells.
\end{abstract}

Keywords: Oxidative Stress, Hematological Neoplasia

\section{Introduction}

\section{Hematological Malignancies}

The term cancer is used to designate more than one hundred different diseases. Evidence indicates that even the most common forms there for a number of cancers, not only environmental influences, as well as hereditary predisposition (Robbins, 2010). The hematological malignancies are diseases caused by uncontrolled proliferation of blood cells or bone marrow failure to produce adequate amount of cells that mainly affect the bone marrow, the site of blood cell formation and occupies the cavity of bones. In it are found the cells that give rise to white blood cells to Red Blood Cells (RBCs or erythrocytes) and platelets (INCA, 2011).

Within this group of diseases called hematological neoplasms are described different clinical syndromes of different etiologies, such as leukemias and lymphomas. Leukemia is a malignancy of white blood cells (leukocytes) clinically and pathologically subdivided in a wide of groups. The first division is in its acute and chronic forms. Acute leukemia is characterized by a rapid increase in the number of immature blood cells, which becomes the bone marrow unable to reproduce healthy blood cells. Further, the chronic form of leukemia is characterized by excessive increase in abnormal cells of mature white blood, taking months or years to progress. The second division concerns the cell type affected by the disorder, thus characterized as lymphoid or myeloid leukemia types (INCA, 2011).

The Acute Lymphoblastic Leukemia (ALL) is the most common childhood cancer, accounting for $25 \%$ of cancers 0-14 years and constitutes $85 \%$ of infant leukemias (Barbosa et al., 2002), already Chronic Lymphocytic Leukemia (CLL) is quite prevalent in adults with age range 67-72 years. The CLL has caused concern, since it is characterized by clonal proliferation and accumulation of malignant $\mathrm{B}$ lymphocytes mature and considered an incurable disease. With this, more and more studies are being conducted to understand the molecular pathogenesis underlying the progression of CLL and its resistance to treatment (Smolewski et al., 2013; Oppezzo and Dighiero, 2013).

The Chronic Myeloid Leukemia (CML) is a myeloproliferative disorder of pluripotent hematologic progenitor cells characterized by excessive proliferation and accumulation of granulocytes and red blood cells and platelets. Ghalaut et al. (2006) predisposes the limitation of studies on this type of cancer and even with only 30 patients in their study emphasized the changes of the lipid profile of these patients after standard chemotherapy, since low concentrations of lipid and lipoproteins are associated with poor prognosis of the disease. Thus, it is proposed that estimation of the lipid 
profile may be helpful in evaluating the response to chemotherapy in CML patients.

Another type of hematologic malignancy are the lymphomas, malignancies originating in the lymph nodes, which are important in fighting infections. For unknown reasons, the number of cases has doubled in the last 25 years. The lymphomas are divided into Hodgkin Lymphoma (HL) and Non-Hodgkin's Lymphoma (NHL) (INCA, 2011). The Non-Hodgkin Lymphoma (NHL), a form of cancer that originates in the lymph nodes of the lymphatic system, can occur at any age, but the incidence of this pathology is higher in young adults between 25 to 30 years. The disease arises when a lymphocyte becomes malignant cell, able to grow uncontrollably and spread. The malignant cell starts to produce identical copies in the lymph nodes. Over time, there is a risk that such malignant cells disseminate to neighboring tissues and, if untreated, to reach other organs. Over the past 50 years, the number of cases remained stable in Brazil, while mortality was reduced by over $60 \%$ since the early $70 \mathrm{~s}$ due to advances in treatment (INCA, 2011). In most cases the etiology of NHL is unknown, although is associated with immune malfunction. Therefore, people who received transplanted treatment with immunosuppressive drugs to prevent rejection of the transplant, patients with severe autoimmune conditions and people infected with HIV have a higher risk for developing NHL (INCA 2011).

\section{Free Radicals, Antioxidants and Oxidative Stress}

Among the several risk factors enrolled in hematological malignancies prognosis, the occurrence of oxidative stress have been recently highlighted. The excessive production of highly reactive free radicals has been investigated in many diseases, especially in cancer. Oxidative stress is characterized by the imbalance between the generation of free radicals ant its scavenging by antioxidants, which can result excessive free radicals, tissue damage and production of toxic or harmful compounds (Burton and Yung, 2011; Robbins, 2010; Schneider and Oliveira, 2004).

Free radicals are all species that have one or more unpaired electrons and this can be centered free electron in an atom of oxygen, hydrogen, nitrogen, carbon, sulfur atoms or transition metals (Junior et al., 2001). These radicals can start chain reactions yielding new radicals and amplifying the ability to produce lesions in proteins, DNA and lipids (Filho, 2011). The organism undergoes constant action of Reactive Oxygen Species (ROS) and Nitrogen (RNS), generated physiologically or pathologically in processes as the mitochondrial respiratory chain, inflammatory processes, and biological dysfunctions or even from food (Halliwell and Gutteridge, 2007). The main generation of free radicals in cells is the reduction of oxygen to water, where the entry of 4 electrons in the oxygen molecule promotes emergence of radical hydrogen peroxide, superoxide anion and the hydroxyl radical (Halliwell and Gutteridge, 2007).

The superoxide anion is produced in the mitochondria or enzymatically by cytoplasmatic enzymes such as xanthine oxidase and cytochrome P450 (Robbins, 2010). The superoxide radical is formed from the addition of an electron (e-) to an oxygen molecule (Schneider and Oliveira, 2004). Superoxide anions are poorly reactive in aqueous solution and are converted to hydrogen peroxide by the enzyme Superoxide Dismutase (SOD), yielding water and molecular oxygen. Hydrogen peroxide also gives rise to the hydroxyl radical, which can strongly react and change any cellular structure that is near and thus influence enzymes, nucleic acids and cellular membranes (Jenkins, 1988).

Free radicals generated during oxidative stress have many cellular targets, but the major are the membrane lipids, causing lipid peroxidation (Schneider and Oliveira, 2004). This oxidation of lipids results in secondary molecules such as alkoxyl, peroxyl, Malondial Dehyde (MDA) and 4-Hydroxynonenal (4-HNE). The latter are electrophilic compounds that can spread throughout the cell, damaging cellular components or interfering with their functioning (Conklin, 2004).

When the production of the reactive species is exacerbated, the cells trigger the antioxidant defenses as a neutralizing mechanism, aiming to block these radicals or inactive them, restoring the balance between pro and antioxidant (Robbins, 2010; Vasconcelos et al., 2007; Halliwell and Gutteridge, 2007).

There are enzymatic and non-enzymatic antioxidant defenses. The enzymatic system includes SOD, Catalase (CAT) and Glutathione Peroxidase (GPx). SOD catalyzes the superoxide radical, while CAT decomposes the hydrogen peroxide (Barreiros and David, 2006). Additional defenses include the glutathione system, where the Glutathione peptide (GSH) acts as a protective mechanism against oxidative stress by converting GSH to oxidized glutathione (Schneider and Oliveira, 2004).

The SOD, CAT and glutathione system, are the first line antioxidants that defend cells against reactive oxygen species and protecting cells from oxidative stress, playing a crucial role in neutralizing the effects of the products of lipid peroxidation (Park et al., 2007; Gago-Dominguez et al., 2007). Other antioxidants includes compounds ingested through regular diet as ascorbic acid (vitamin C), $\alpha$-tocopherol (vitamin E) and $\beta$-carotene or synthesized by the human body, such as 
uric acid, bilirubin, sex hormones, melatonin and ceruloplasmin (Schneider and Oliveira, 2004).

\section{The Oxidative Status of Patients with Hematological Neoplasms}

Disturbances in the redox homeostasis are commonly associated with the arising of tumor cells. However, the main question that remains unclear is about the role of oxidative status in the pathology of the human cancer. Some studies have provided interesting evidences. For solid tumors, such as breast cancer, it is established that oxidative stress have been indicated as a major mediator of cancer staging progression (Panis et al., 2011). On the other hand, evidences point that metabolites derived from the lipid peroxidation process can represent a protective mechanism in breast cancer (Gago-Dominguez et al., 2007).

Regarding hematological neoplasms, some recent studies have characterized the oxidative status of patients and reported as follows. Kelkel et al. (2010) states that the imbalance between the production of Oxygen/Nitrogen-derived free Radicals (ROS/RNS) and antioxidants, featuring oxidative stress occurence in patients with different types of leukemia, confirming that the leukemic cells can produce more ROS than normal non-leukemic cells.

Additionally, it is also known that oxidative stress is closely involved with the occurrence of the toxicity of chemotherapy. Research has shown that patients with solid tumors undergoing treatment with chemotherapeutic drugs doxorubicin and paclitaxel have immediate treatment and oxidative damage of the blood cells with the development of anemia (Panis et al., 2011) systemic oxidative stress. In addition, chemotherapy causes damage to various organs of patients who are in continuous treatment with this anticancer causing significant side effects mediated by oxidative stress (Campos et al., 2012). The same seems to occur in the case of hematological malignancies, since previous studies have shown that oxidative stress is closely linked to the development of chemoresistance in adult patients with chronic myeloid leukemia (Pizzatt et al., 2012).

Ghalaut et al. (1999) showed the glutathione in lymphocytes of patients with acute leukemia before and after treatment and concluded that lymphocytic glutathione levels were higher in the active phase of disease than in the remission phase, suggesting that the glutathione level could act as a marker of leukemic activity and may help to predict onset of relapse.

Zhou et al. (2010) analyzed patients with Acute Myeloid Leukemia (AML) some markers of oxidative stress, such as the Total Antioxidant Capacity (T-AOC), the activities of SOD, glutathione peroxidase, xanthine oxidase and MDA levels in order to evaluate the recurrence of disease and its relation with the oxidative status of patients. The results of this investigation indicated that the relapse of AML is associated with alterations in antioxidant defenses, suggesting a correlation between oxidative stress and the rate of AML relapse as a potential prognostic marker.

Another study conducted by Devi et al. (2000) explored the antioxidant system and its correlation with leukemias features. It was suggested that patients prior to therapy with different types of leukemia presented increased levels of oxidative stress on its leukocytes, characterized by the imbalance of several parameters such as alterations in superoxide anion and hydrogen peroxide, plasma malondialdehyde levels, red cell Copper Zinc Superoxide Dismutase (Cu-Zn SOD) and Glutathione Peroxidase (GSH-PX). Although the oxidative stress occurrence in leukocytes, no differences wer found among the distinct types of leukemias.

It has been widely demonstrated that the action of reactive oxygen species on proteins increase the formation of Carbonyl Groups (CG). In order to quantify the oxidative stress in patients with B-Chronic Lymphocytic Leukemia (B-CLL), Musolino et al. (2011) investigated the serum levels of CG demonstrated a significant increase of CG in B-CLL patients, suggesting the occurrence of systemic oxidative changes in such disease.

Another point of interest is the studies concerning the Acute Lymphocytic Leukemia (ALL). Enhanced levels of systemic oxidative stress have been reported in ALL patients, in association with DNA oxidative injuries, when comparing with healthy age-matched individuals (Devi et al., 2000). Significant reduction of enzymatic antioxidants has been also found in ALL (Senturker et al., 1997). Modifications of the redox status are pointed as putative enrolled with the development of chemoresistant phenotypes. In vitro studies indicate that chemoresistant ALL cells may be associated with the lacking of response to oxidative stress-driven death signals, in association with enhancement in the antioxidant capacity of such cancer cells (Goto et al., 2007).

\section{Conclusion}

Studies regarding the relationship between oxidative changes and hematological malignancies are still sparse. Most of the studies points to the occurrence of an imbalance mediated by excessive free radical production, with impaired production of antioxidants. The enhancement of the antioxidant content of cancerous cells may also be a key event to the acquisition of chemoresistance of leukemic cells. 
Further studies are necessary to establish the causeconsequence relationship concerning oxidative stress and hematological malignancies, especially regarding the mechanisms enrolled in the parameters that determine poor disease prognosis.

\section{Author's Contributions}

All authors equally contributed in this work.

\section{Ethics}

This article is original and contains unpublished material. The corresponding author confirms that all of the other authors have read and approved the manuscript and no ethical issues involved.

\section{References}

Barbosa, C.M.P.L., C. Nakamura, M.T. Terreri, M.L.M. Lee and A.S. Petrilli, 2002. Manifestações músculoesqueléticas como apresentação inicial das leucemias agudas na infância. J. de Pediatria, 78: 481-4. DOI: 10.2223/JPED.903

Barreiros, A.L.B.S., J.M. David and J.P. David, 2006. Estresse oxidativo: Relaçãoentre geração de espécies reativas e defesa do organismo. Quim. Nova, 29: 113-123. DOI: $10.1590 / \mathrm{S} 0100-40422006000100021$

Burton, G.J. and H.W. Yung, 2011. Endoplasmic reticulum stress in the pathogenesis of early-onset pre-eclampsia. Pregnancy Hypertens, 1: 72-78. DOI: $10.1016 /$ j.preghy.2010.12.002

Campos, F.C., P. Carolina, T. De Rossi, V.J. Victorino and A.O.C. Armani et al., 2012. Aspects related to oxidative stress-mediated toxicity of doxorubicin. Applied Cancer Res., 32: 25-29.

Conklin, K.A., 2004. Free Radicals: The pros and cons of antioxidants. Am. Soc. Nutrit. Sci. J. Nutr., 134: 3201-3204.

Devi, G.S., M.H. Prasad, I. Saraswathi, D. Raghu and D.N. Rao et al., 2000. Free radicals antioxidant enzymes and lipid peroxidation in different types of leukemias. Clin. Chim. Acta., 293: 53-62. DOI: 10.1016/S0009-8981(99)00222-3

Filho, G.B., 2011. Bogliolo Patologia. $8^{\circ}$ edição. Editora Guanabara Koogan LTDA. Rio de Janeiro-RJ. 1501.

Gago-Dominguez, M., X. Jiang and E. Castelao, 2007. Lipid peroxidation, oxidative stress genes and dietary factors in breast cancer protection: A hypothesis. Breast Cancer Res., 9: 201-212. DOI: 10.1186/bcr1628

Ghalaut, V.S., M.B.P. Sunita and P.S. Ghalaut, 2006. Alteration in lipid profile in patients of chronic myeloid leukemia before and after chemotherapy. Clin. Chim. Acta., 366: 239-242.

DOI: $10.1016 /$ j.cca.2005.10.022
Ghalaut, V.S., S. Kharb, P.S. Ghalaut and A. Rawal, 1999. Lymphocyte glutathione levels in acute leukemia. Clin. Chim. Acta., 285: 85-89.

DOI: 10.1016/S0009-8981(99)00109-6

Goto, Y., S. Otani and A.A. Grace, 2007. The yin and yang of dopamine release: A new perspective. Neuropharmacology, 53: 583-587. DOI: $10.1016 /$ j.neuropharm

Halliwell, B. and J.M.C. Gutteridge, 2007. Free Radicals in Biology and Medicine. 4th Edn., New York, Oxford University Press, ISBN-10: 019856869X, pp: 704.

INCA, 2011. Estimativa 2012 de incidência do câncer no Brasil Brasil/Instituto Nacional deCâncer José Alencar Gomes da Silva, Coordenação Geral de Ações Estratégicas, Coordenação de Prevenção e Vigilância. INCA, Rio de Janeiro.

Jenkins, R.R., 1988. Free radical chemistry relationship to exercise. Sports Med., 5: 156-70. DOI: 10.2165/00007256-198805030-00003

Junior, L.R., N.F. Hoehr and A.P. Vellasco, 2001. Sistema antioxidante envolvendo o ciclo metabólico da glutationa associado a métodos eletroanalíticos na avaliação do estresse oxidativo. Quim. Nova, 24: 112-119. DOI: 10.1590/S0100-40422001000100019

Kelkel, M., C. Jacob M. Dicato and M. Diederich, 2010. Potential of the Dietary antioxidants resveratrol and curcumin in prevention and treatment of hematologic malignancies. Molecules, 15: 7035-7074. DOI: 10.3390/molecules 15107035

Musolino, C., A. Allegra, A. Alonci, A. Saija and S. Russo et al., 2011. Carbonyl group serum levels are associated with CD38 expression in patients with B chronic lymphocytic leukemia. Clin. Biochem., 44: 1487-1490. DOI: 10.1016/j.clinbiochem.2011.08.1149

Oppezzo, P. and G. Dighiero, 2013. Role of the B-cell receptor and the microenvironment in chronic lymphocytic leukemia. Blood Cancer J. DOI: $10.1038 /$ bcj.2013.45

Panis, C., A.C.S.A. Herreira and V.J.V. Colaboradores, 2011. Oxidative stress and hematological profiles of advanced breast cancer patients subjected to paclitaxel or doxorubicin chemotherapy. Breast Cancer Res. Treat., 133: 89-97. DOI: 10.1007/s10549-011

Park, E.M., N. Ramnath, G.Y. Yang, J.Y. AHN and Y. Park Lee et al., 2007. High SOD and low GPX activities in RBC predict susceptibility of lung cancer patients to radiation pneumonitis. Free Radic. Biol. Med., 42: 280-287. DOI: 10.1016/j.freeradbiomed.2006.10.044

Pizzatt, L., C. Panis, G. Lemos, M. Rocha and R. Cecchini et al., 2012. Label-free MSE proteomic analysis of chronic myeloid leukemia bone marrow plasma: Disclosing new insights from therapy resistance. DOI 10.1002/pmic.201200066 
Robbins, K.C., 2010. Patologia Estrutural Funcional. 8th Ed., Elsevier.

Schneider, C.D. and A.R. Oliveira, 2004. Radicais livres de oxigênio e exercício: Mecanismos de formação e adaptação ao treinamento físico. Rev. Bras. Med. Esporte.

DOI: $10.1590 / \mathrm{S} 1517-86922004000400008$

Senturker, S., B. Karahalil, M. Inal, H. Yilmaz and H. Müslümanoglu et al., 1997. Oxidative DNA base damage and antioxidant enzyme levels in childhood acute lymphoblastic leukemia. FEBS Lett., 416: 286-290.

DOI: $10.1016 / \mathrm{S} 0014-5793(97) 01226-\mathrm{X}$
Smolewski, P., M. Witkowska, A. KoryckaWoBowiec, 2013. New Insights into biology, prognostic factors and current Therapeutic strategies in chronic lymphocytic leukemia. ISRN Oncol. DOI: 10.1155/2013/740615

Vasconcelos, E.S.D., C.D. Cruz, L.L. Bhering, M.F. Ribeiro and R. Júnior, 2007. Método alternativo para análise de agrupamento. Pesquisa Agropecuária Bras. DOI: 10.1590/S0100-204X2007001000008

Zhou, F.L., W.G. Zhang, Y.C. Wei, S. Meng and G.G. Bai et al., 2010. Involvement of oxidative stress in the relapse of acute myeloid leukemia. J. Biol. Chem., 285: 15010-15015. DOI: 10.1074/jbc.M110.103713 